\title{
Fooled by Rounding
}

\author{
John Mason ${ }^{1}$ (D) $\cdot$ Rina Zazkis ${ }^{2}$
}

Published online: 8 October 2019

(C) The Author(s) 2019

\begin{abstract}
For any convex quadrilateral, joining each vertex to the mid-point of the next-but-one edge in a clockwise direction produces an inner quadrilateral (as does doing so in a counter-clockwise direction). In many cases, a dynamic geometry measurement of the ratio of the area of the outer quadrilateral to the area of the inner one appears to be $5: 1$. It turns out, however, that this is due to rounding. We generalise the construction by replacing mid-points by more general ratios, finding the maximum and minimum values of the area ratio and determining the conditions on the original quadrilateral that achieve those two extremes.
\end{abstract}

Keywords Dynamic geometry software $\cdot$ Computer algebra software $\cdot$ Geometry-algebra interplay $\cdot$ Being misled by missing decimal places $\cdot$ Convex quadrilaterals $\cdot$ Ratios of areas

\section{Introduction}

Given a convex quadrilateral, form the mid-points of the edges and then join them to the vertices in cyclic order, as shown in Fig. 1.

In dynamic geometry software, the ratio of the area of the outer to the area of the inner quadrilateral is often given to be $5: 1$ over a range of positions for $\mathrm{W}$ - so much so that it is tempting to conjecture that the ratio will always be that (both authors have been so tempted, independently). However, when the software is induced to show as many decimal places as possible, it turns out that the ratio differs from 5:1 in the third or fourth decimal digit over quite a large range of choices of $\mathrm{W}$. When people use dynamic geometry software to construct an example, their starting configurations are

\footnotetext{
${ }^{1}$ We use the British English term trapezium, meaning a quadrilateral with at least one pair of opposite sides parallel.
}

John Mason

1 Open University Milton Keynes and University of Oxford, Oxford, UK

2 Simon Fraser University, BC, Canada 



Fig. 1 Clockwise and counter-clockwise orientations for the mid-point construction

often quite close to being trapezia ${ }^{1}$ (trapezoidal), for which the ratio really is exactly $5: 1$, and small variations in $\mathrm{W}$ make little difference in the ratio.

The aim here is to show two things: that the ratio of the area of the outer quadrilateral to the area of the inner quadrilateral is always at least $5: 1$, with equality only when the inner quadrilateral is a trapezium (trapezoid), and that the ratio is at most $6: 1$, with this equality only when the quadrilateral degenerates into a triangle with $\mathrm{W}$ coinciding with either X or Z. The situation is then generalised.

\section{Remarks about Being Fooled by Rounding}

When students use a calculator to compute the ratio of consecutive terms from a Fibonacci sequence, they often conclude that the ratio is constant, but this is due to the limited number of decimal places used on their calculator. Even though well aware of this phenomenon, it is still easy to be caught similarly in fresh situations, which is what happened to us with the outer to inner area ratio. Even when the ratio shifts to 5.01:1, say, this is perhaps more likely to be dismissed as a rounding error than treated as a genuine counter-example to the constant-ratio conjecture. After all, when there is a whole region of invariance in a DGS phenomenon, it is likely to be universal.

\section{Special Case: Mid-Point Reasoning}

Let $\mathrm{X}, \mathrm{Y}$ and $\mathrm{Z}$ be three vertices of a convex quadrilateral in cyclic order, and $\mathrm{W}$ the fourth point. By translation, $Y$ can be taken as the origin. By rotation and scaling in skew directions, $\mathrm{X}$ and $\mathrm{Z}$ can be taken to be $[2,0]^{2}$ and $[0,2]$ and these affine actions do not affect the ratio of areas. The use of 2 is in order to avoid fractions when calculating mid-points. Let $\mathrm{W}$ then be $[2 a, 2 b]$. Alternatively, but equivalently, treat $\mathrm{X}-\mathrm{Y}$ and $\mathrm{Z}-$ $\mathrm{Y}$ as basis vectors and then $\mathrm{W}=2 a(\mathrm{X}-\mathrm{Y})+2 b(\mathrm{Z}-\mathrm{Y})$.

These 'affine-simplified' co-ordinates make the algebra a great deal simpler. However, in order to combine algebra with general DGS diagrams, it is useful to construct a

\footnotetext{
${ }^{1}$ We use the British English term trapezium, meaning a quadrilateral with at least one pair of opposite sides parallel.

${ }^{2}$ Due to the many brackets in the on-coming algebraic expressions, in this piece co-ordinates are indicated using square brackets $-[a, b]-$ rather than round ones $-(a, b)$.
} 
procedure which converts DGS co-ordinates into affine-simplified co-ordinates (so as to check geometrical relationships algebraically), together with its inverse, in order to convert affine-simplified co-ordinates into DGS co-ordinates (so as to display and check the results of algebraic calculations geometrically).

Restricting the outer quadrilateral to being convex is achieved through requiring $a, b$ and $a+b-1$ to be non-negative (see Fig. 2).

The applet Outer to inner quadrilateral area ratios (Mason, 2019) provides displays for the more general situation, as well as for this initial special case.

The relation between the two orientations is that interchanging the first and second co-ordinates, and interchanging $a$ and $b$, converts the first clockwise orientation diagram in Fig. 2 into the other orientation. Thus, it is sufficient to work only with the first orientation.

The mid-points of the edges of the quadrilateral are

$$
\begin{aligned}
& \mathrm{M}_{\mathrm{WX}}=\frac{(W+X)}{2} \\
& \mathrm{M}_{\mathrm{XY}}=\frac{(X+Y)}{2} \\
& \mathrm{M}_{\mathrm{YZ}}=\frac{(Y+Z)}{2} \\
& \mathrm{M}_{\mathrm{ZW}}=\frac{(Z+W)}{2}
\end{aligned}
$$

So, in affine-simplified co-ordinates,

$$
\begin{gathered}
\mathrm{M}_{\mathrm{WX}}=[1+a, b] \\
\mathrm{M}_{\mathrm{XY}}=[1,0] \\
\mathrm{M}_{\mathrm{YZ}}=[0,1] \\
\mathrm{M}_{\mathrm{ZW}}=[a, 1+b]
\end{gathered}
$$
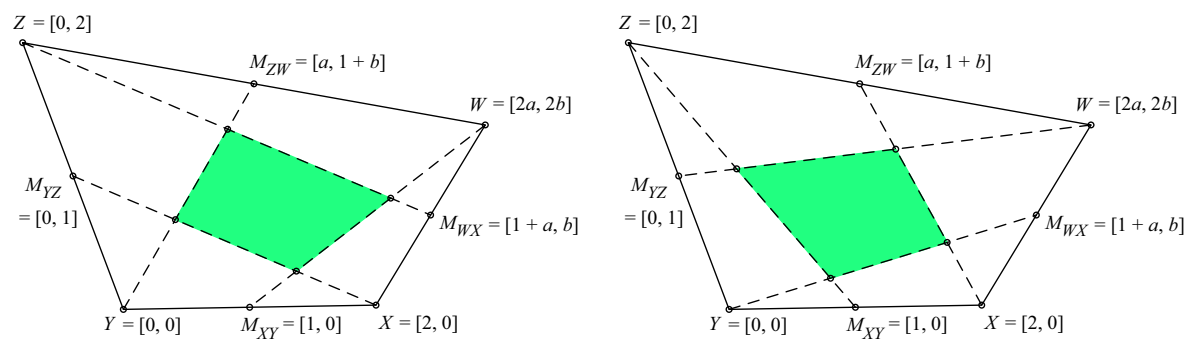

Fig. 2 Affine-simplified co-ordinates 
The vertices of the inner quadrilateral can then be found (the calculations are omitted due to not being edifying; they will be done more generally shortly). The inner quadrilateral is a trapezium in the first orientation, either when the segments $Z M_{W X}$ and $\mathrm{XM}_{\mathrm{YZ}}$ are parallel or when $\mathrm{WM}_{\mathrm{XY}}$ and $\mathrm{YM}_{\mathrm{ZW}}$ are parallel. In terms of affinesimplified co-ordinates, these conditions are expressed algebraically as $a+2 b-3=0$ or $2 a-b-1=0$, respectively. Corresponding conditions for the second orientation can be produced by interchanging co-ordinates and reversing $a$ and $b$.

Because of what happens in the area calculations, it is convenient to specify $T=(a+$ $2 b-3)(2 a-b-1)$, noting that, when both factors are zero, the inner quadrilateral is a parallelogram and $a=b=1$.

The locus of positions of $\mathrm{W}$ that make the inner quadrilateral a trapezium, while still preserving the convexity of the outer quadrilateral, is shown in Fig. 3 using two finedotted lines. Notice that because of the requirement for convexity, one is a segment bounded by the extended edges $\mathrm{YX}$ and $\mathrm{YZ}$ of the quadrilateral, while the other is a ray with its vertex on the diagonal XZ. These two lines are parallel to the pair of parallel edges of the inner trapezium. The intersection point is the position for $\mathrm{W}$ that makes the inner quadrilateral a parallelogram. The outer quadrilateral is then also a parallelogram in general, but a rhombus in affine-simplified co-ordinates.

The ratio of the areas of the outer to the inner quadrilateral was found initially by using dynamic geometry software, then gazing at the algebra trying to make use of the trapezium conditions. The lower bound of 5:1 was anticipated to occur when $a=b=1$ (and the inner quadrilateral is a parallelogram). The upper bound of 6:1 was found by taking a limiting case in which the quadrilateral degenerates into a triangle when $\mathrm{W}$ coincides with either X or Z $(a=1$ and $b=0$ or $a=0$ and $b=1)$, which the DGS suggests is where the maximum occurs (see Fig. 4).

Using the anticipated bounds, the algebra involves large polynomials in $a$ and $b$ which can be re-expressed usefully as follows:

$$
\rho=\frac{\text { Area }_{\text {outer }}}{\text { Area }_{\text {inner }}}=5+\frac{T^{2}}{T^{2}+4 S}=6-\frac{4 S}{T^{2}+4 S}
$$

where $S=S_{1} S_{2}$ with $S_{1}=a b+2 b^{2}+4 a-2$ and $S_{2}=4 a b+2 a^{2}-3 a+b+1$.

With hindsight, it is easy to re-express these as:

$$
S_{1}=a b+2(b-1)^{2}+4(a+b-1) \geq 0 \text {, since } a+b \geq 1 \text { and } a \geq 0 \text { and } b \geq 0 \text {, }
$$
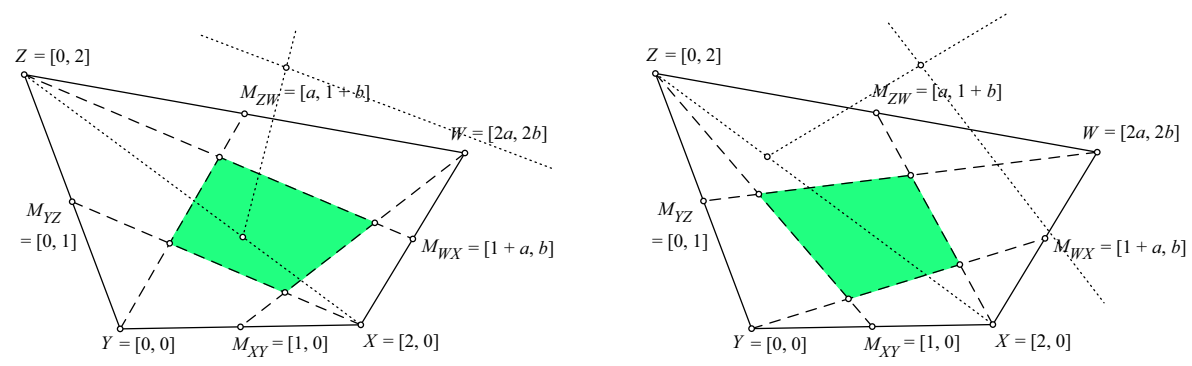

Fig. 3 Loci of $\mathrm{W}$ for trapezia while preserving convexity 



Fig. 4 Positions of $\mathrm{W}$ with the maximum ratio while preserving convexity

and

$$
S_{2}=2(a-1)^{2}+4 a b+(a+b-1) \geq 0 \text {, for the same reasons. }
$$

Because $S_{1}$ and $S_{2}$ are both non-negative when $a, b$ and $a+b-1$ are all nonnegative, 5:1 and 6:1 are the lower and upper bounds for the ratio of the area of the outer quadrilateral to the area of the inner quadrilateral, when the outer quadrilateral is convex.

The expressions for $\rho$ were the result of considerable exploration, including eventually conjecturing that the denominator might be expressed in terms of $T$ and $S$. Only later did it become obvious how to find them logically, as will emerge shortly in the more general setting.

The equations $S_{1}=0$ and $S_{2}=0$ represent conics and, as can be seen from the expression of the ratio, turn out to be the loci of $\mathrm{W}$ for which the algebraic area ratio is 6:1. They are shown in Fig. 5. However, the only values of $a$ and $b$ that lie on this locus, and which also maintain a convex quadrilateral, are when $a=1$ and $b=0$, or when $a=0$ and $b=1$, as was shown in Fig. 4. These are limiting positions for W.

\section{Notes about Relationships}

Geometrically, when the inner quadrilateral is a parallelogram, the ratio of 5:1 can be seen fairly directly, as shown in Fig. 6.

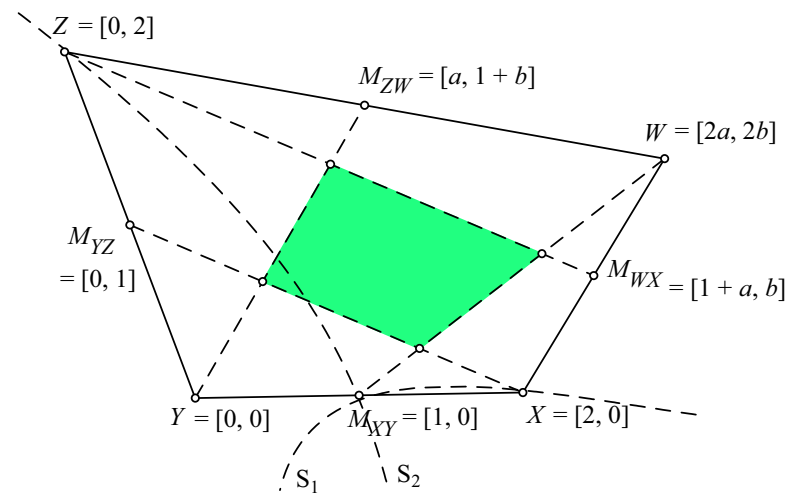

Fig. 5 Loci of $\mathrm{S}_{1}=0$ and $\mathrm{S}_{2}=0$ shown dashed 

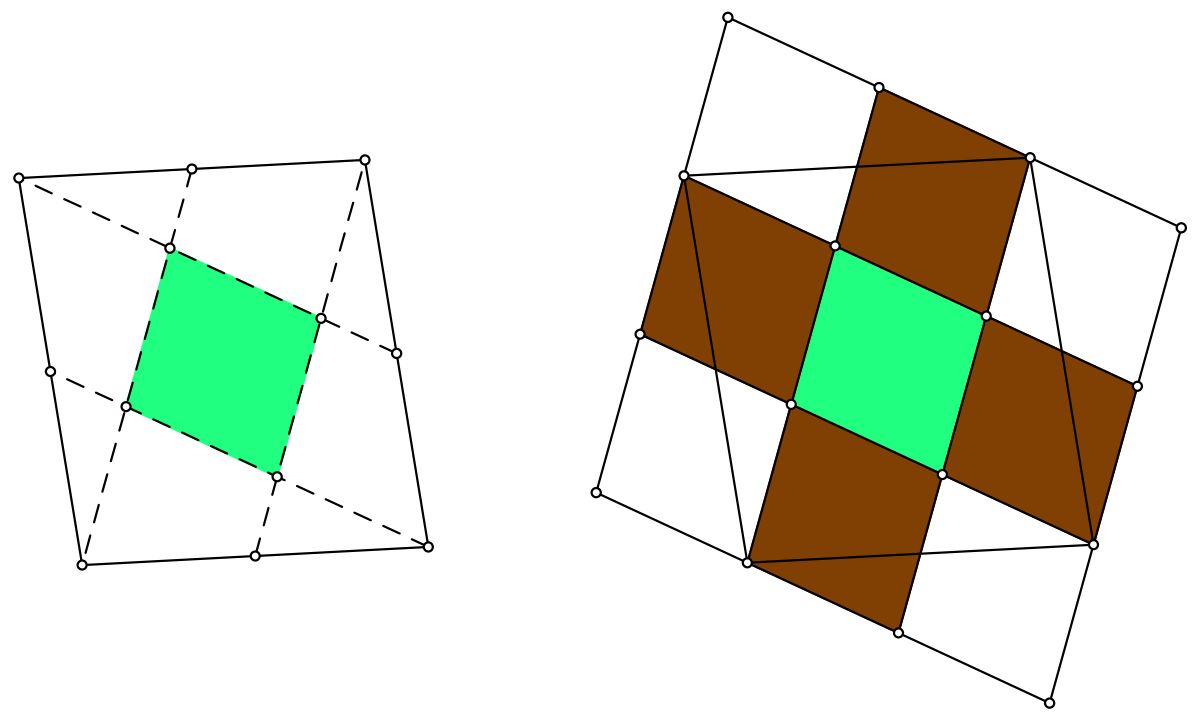

Fig. 6 Minimum ratio achieved for parallelogram seen as an area calculation (left) and as two grids superimposed (right)

Using the fact of the mid-point construction

$$
\frac{\text { Area }_{\text {outer }}}{2}=(4 A+D)+(4 B+C)=(4 C+A)+(4 D+B)
$$

where each bracket is half of the area of half of the whole outer quadrilateral. The area covered twice is $A+B+C+D$, and the area not covered is the Inner Area, from which it follows by the carpet theorem (Bogomolny, 2018) or by simple algebra, that

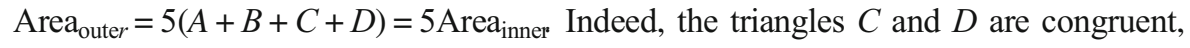
as are the triangles $A$ and $B$ (Fig. 6, left side). Two superimposed grids depict these congruences geometrically 'without words', in Fig. 6 (right side).

When the inner quadrilateral is a trapezium but not a parallelogram, there is only one pair of parallel lines forming the trapezium.

Using $\mathrm{C}$ and $\mathrm{D}$ to label the areas as shown in Fig. 7 (left side), it is still the case that Area $_{\text {inner }}=A+B+C_{1}+D$. Drawing in parallel lines in Fig. 7 (right) side produces two
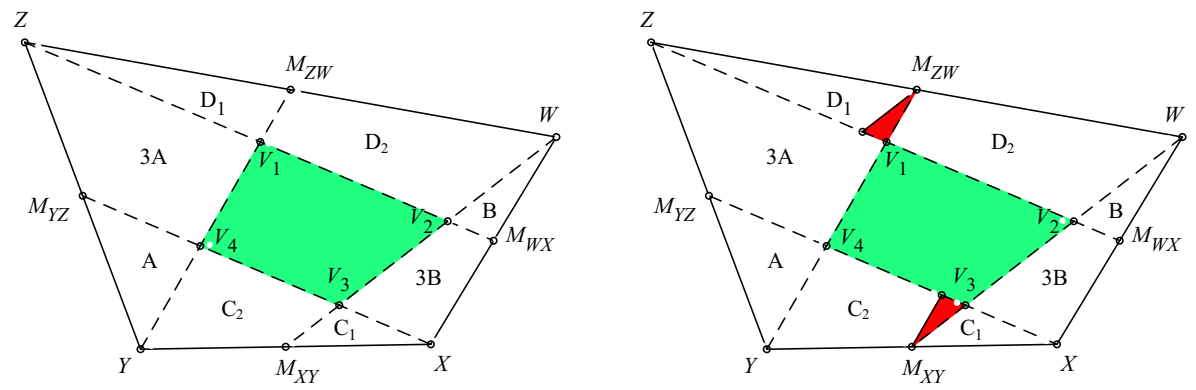

Fig. 7 Labelling areas and forming the shaded triangles with parallel lines 
small triangles with parallel sides which are therefore similar. The shaded triangles are congruent according to the algebra, but it is not obvious how to prove this synthetically.

\section{Some Generality: Edges Divided in the Ratio $\mu:(1-\mu)$}

The next step was to generalise the mid-point construction to dividing the edges in the ratio $\mu:(1-\mu)$ in cyclic order around the quadrilateral. Since this case is subsumed in the following more general result, details are omitted. The minimum and maximum area ratios for the clockwise construction turned out to be:

$\frac{1-\mu^{2}}{(1-\mu)^{2}}$ and $\frac{1-\mu+\mu^{2}}{(1-\mu)^{3}}$ which, in the case of $\mu=\frac{1}{2}$, have the values 5 and 6 respectively.

The counter-clockwise values are obtained by replacing $\mu$ by $1-\mu$.

\section{General Theorem: Opposite Edges Divided in Given Ratios}

Given a convex quadrilateral X, Y, Z, W, in cyclic order, divide one pair of opposite edges in the ratio $\mu:(1-\mu)$ and the other pair in the ratio $\nu:(1-\nu)$, both in cyclic order. Join each vertex to the division-point on the next-but-one edge clockwise, and do the same counter-clockwise. This produces two inner quadrilaterals. Denote by $\rho$ the ratio of the area of the outer quadrilateral to the area of the inner quadrilateral.

Then, for the clockwise orientation, $\frac{1+\mu \nu}{(1-\mu)(1-v)} \leq \rho \leq \max \left(\frac{1-\mu(1-\nu)}{(1-\mu)(1-\nu)^{2}}, \frac{1-\nu(1-\mu)}{(1-\mu)^{2}(1-\mu)}\right)$.

For the counter-clockwise orientation, the bounds are found by replacing $\mu$ by $1-\nu$, and $\nu$ by $1-\mu$ so

$$
\frac{1+(1-\mu)(1-v)}{\mu v} \leq \rho \leq \max \left(\frac{1-\mu(1-v)}{\mu v^{2}}, \frac{1-v(1-\mu)}{\mu^{2} v}\right) .
$$

\section{Development and Proof}

Start with simplifying the co-ordinates, without loss of generality, using affine transformations. Since $\mu$ and $\nu$ are used, it is sensible to use co-ordinates [0, 0], [0, 1], [1, 0], and $[a, b]$ for the vertices, as shown in Fig. 8, in the two orientations.

Convexity requires the conditions that $a, b$ and $a+b-1$ be non-negative.

The division points are given by:

$$
\begin{gathered}
\mathrm{M}_{\mathrm{WX}}=(1-\mu) \mathrm{W}+\mu \mathrm{X}=[(1-\mu) a+\mu,(1-\mu) b] \\
\mathrm{M}_{\mathrm{ZW}}=\nu \mathrm{W}+(1-\nu) \mathrm{Z}=[\nu a,(1-\nu)+\nu b] \\
\mathrm{M}_{\mathrm{YZ}}=(1-\mu) \mathrm{Y}+\mu \mathrm{Z}=[0, \mu]
\end{gathered}
$$



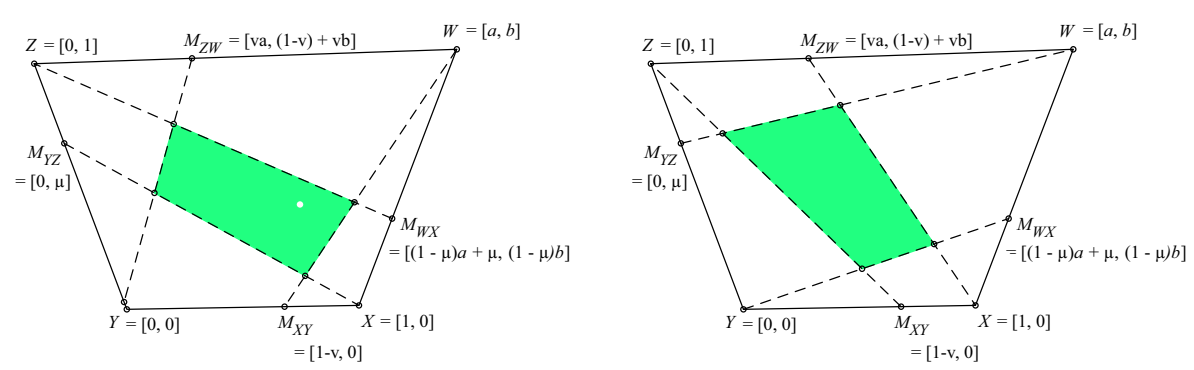

Fig. 8 Inner quadrilaterals formed by clockwise and counter-clockwise $[\mu, v]$ divisions

$$
\mathrm{M}_{\mathrm{XY}}=(1-\nu) \mathrm{X}+\nu \mathrm{Y}=[1-\nu, 0]
$$

Since the two orientations are related by interchanging first and second co-ordinates, interchanging $a$ and $b$, and interchanging $\mu$ with $1-\nu$, (this will be referred to as the interchange), it is again sufficient to deal with the clockwise orientation only. It is, however, useful to carry both sets of calculations forward in a CAS for oneself for comparison later.

A reasonable conjecture is that the trapezia condition will still give the minimum area ratio. The condition for the inner quadrilaterals to be trapezia is that $\mu \nu \mathrm{T}=0$, where $T=(\mu(a-1)+(b-1))((a-1)-\nu(b-1))$. This locus is displayed in Fig. 9 for both orientations. Note that again the locus of $W$ (which forces the inner quadrilateral to be a trapezium) consists of lines through $[1,1]$ parallel to the pair of parallel lines forming the trapezium.

The corresponding conditions for the counter-clockwise orientation are found by making the interchange. The relevance of expressing these in terms of $a-1$ and $b-1$ will appear later.

Sticking with the clockwise orientation, the equations of the lines forming the inner quadrilateral can be calculated, and from them the vertices of the clockwise inner quadrilateral can be found with the help of a CAS (see Fig. 10):

$$
\begin{aligned}
\mathrm{zmwx} & =\operatorname{Line}\left(\mathrm{Z}, \mathrm{M}_{\mathrm{WX}}\right), \text { whose equation is }:(y-1)(\mu+(1-\mu) a)+x(1-(1-\mu) b) \\
& =0
\end{aligned}
$$
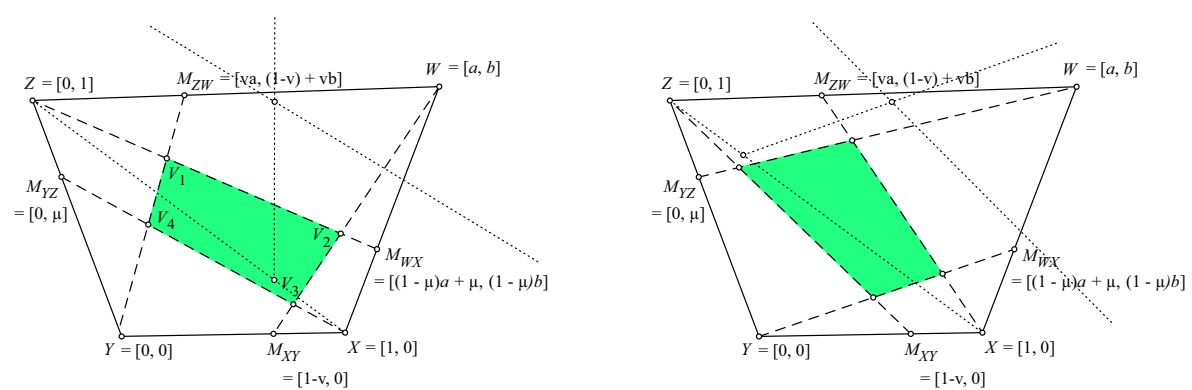

Fig. 9 Showing loci of points $W$ for which the inner quadrilateral is a trapezium 




Fig. 10 Affine-simplified co-ordinates and inner vertex labels in clockwise orientation

$\mathrm{ymzW}=\operatorname{Line}\left(\mathrm{Y}, \mathrm{M}_{\mathrm{ZW}}\right)$, whose equation is : $y \nu a-x(1-\nu(1-b))=0$;

xmyz $=$ Line $\left(\mathrm{X}, \mathrm{M}_{\mathrm{YZ}}\right)$, whose equation is $: y+(x-1) \mu=0$;

wmyz $=$ Line $\left(\mathrm{W}, \mathrm{M}_{\mathrm{YZ}}\right)$, whose equation is : $(y-b) a+(x-a)(\mu-b)=0$.

$V_{1}=y m z w \wedge z m w x=\left[\frac{v a(a+\mu-\mu a)}{\mu \nu\left(a+b^{-1}\right)+(a+\mu-\mu a)}, \frac{(1+v(b-1))\left(a+\mu^{-\mu a}\right)}{\mu \nu\left(a+b^{-1}\right)+\left(a+\mu^{-\mu}\right)}\right]$

$V_{2}=z m w x \wedge w m x y$

$=\left[\frac{(a+\mu-a \mu)(a+b-1+\nu(1-b))}{(a+b-1)+\nu(1-b+b \mu)}, \frac{b((a+b-1)(1-\mu)+b(1-\nu+\mu \nu))}{(a+b-1)+\nu(1-b+b \mu)}\right]$

$V_{3}=w m x y \wedge x m y z=\left[\frac{(a \mu+\mu(\nu-1))+b(1-v)}{a \mu+\mu(\nu-1)+b}, \frac{b \mu \nu}{a \mu+\mu(\nu-1)+b}\right]$

$V_{4}=x m y z \wedge y m z w=\left[\frac{a \mu \nu}{a \mu \nu+1+\nu(b-1)}, \frac{\mu(1+\nu(b-1))}{a \mu \nu+1+\nu(b-1)}\right]$

Algebraic manipulation can be eased by constructing a procedure in a CAS which calculates the lines and their pairs of intersections, and then applying it four times. This makes it easy to carry forward in parallel the calculations associated with the other orientation. 
The ratio of the outer to inner areas, denoted by $\rho$ as a function of $\mu$ and $\nu$, can be found (a computer algebra system is again recommended!). However, making sense of the multiple lines of algebraic output requires some judicious conjecturing. In retrospect, much later, it was straightforward to conjecture that the minimum ratio occurs when the inner quadrilateral is a parallelogram, which means $W=[1,1]$ and then to find useful expressions for the ratio. The first conjecture is that:

$$
\rho_{\text {min }}=\frac{1+\mu v}{(1-\mu)(1-v)} .
$$

Subtracting $\rho_{\min }$ from the area ratio $\rho$ and factoring reveals the numerator of the residue to be $-\mu \nu^{2} T^{2}$, which is confidence-inspiring, since the minimum should be achieved when the inner quadrilateral is a trapezium so that the residual term will be 0 , though the negative sign is a trifle worrying. The unexpected negative sign also appears in the denominator, but it takes some thinking to locate a convenient form for this denominator. Proceed first to consider the maximum.

It is likely that the maximum occurs when $\mathrm{W}=[1,0]$, or $\mathrm{W}=[0,1]$. It turns out that, in general, these are different:

$$
\rho_{\max 1}=\frac{1-\mu(1-v)}{(1-\mu)^{2}(1-v)} \text { and } \rho_{\max 2}=\frac{1-v(1-\mu)}{(1-\mu)(1-v)^{2}} .
$$

Their difference is $\rho_{\max 1}-\rho_{\max 2}=\frac{\mu \nu(\mu-v)}{(1-\mu)^{2}(1-v)^{2}}$, which means that $\rho_{\max 1}$ is conjectured to be the maximum when $\mu \geq \nu$, otherwise the conjectured maximum is $\rho_{\max 2}$. The reverse will be the case in the other orientation.

Subtracting $\rho$ from each of these potential maxima, and factoring, reveals the numerators to be $-\mu^{2} \mathrm{v} S$ and $-\mu v^{2} S$, where $S$ is the product of two quadratics in $a$ and $b$, one of which is zero when $\mathrm{W}=\mathrm{Z}$, that is, when $a=0$ and $b=1$. Thus, negative signs appear in both numerator and denominator and, consequently, can be removed. Simple algebra (if only it had been thought of in the beginning instead of much later!), making use of the difference between the conjectured maximum and the conjectured minimum enables the ratio of outer to inner area to be presented as:

$$
\begin{gathered}
\rho=\frac{\text { Area }_{\text {outer }}}{\text { Area }_{\text {inner }}}=\frac{1+\mu \nu}{(1-\mu)(1-\nu)}+\left(\frac{\mu}{1-\mu}\right)\left(\frac{\nu}{1-\nu}\right) \frac{\mu \nu T^{2}}{(1-\mu) \nu T^{2}+S} \\
=\frac{1-\mu(1-\nu)}{(1-\mu)^{2}(1-\nu)}-\left(\frac{\mu}{1-\mu}\right)^{2}\left(\frac{\nu}{1-\nu}\right) \frac{S}{(1-\mu) \nu T^{2}+S} \\
=\frac{1-v(1-\mu)}{(1-\mu)(1-\nu)^{2}}-\left(\frac{\mu}{1-\mu}\right)\left(\frac{\nu}{1-\nu}\right)^{2} \frac{(\nu-\mu) T^{2}+S}{(1-\mu) \nu T^{2}+S} \\
=\frac{1-v(1-\mu)}{(1-\mu)(1-\nu)^{2}}-\left(\frac{\mu}{1-\mu}\right)\left(\frac{\nu}{1-\nu}\right)^{2} \frac{R}{\mu(1-\nu) T^{2}+R}
\end{gathered}
$$


where $S=S_{1} S_{2}$ and

$$
\begin{gathered}
S_{1}=(a+b-1)+a b \mu \nu+(b-1)^{2} \\
S_{2}=(a-1)^{2} \mu(\mu \nu+\nu+1-\mu)+a b(\mu \nu(\mu+1-\nu)+\nu+1-\mu)+(b-1)^{2} \nu(\mu-\nu)+ \\
(a+b-1)\left(\mu^{2} \nu^{2}+\mu \nu(\nu+1-\mu)+\mu-\nu\right)
\end{gathered}
$$

It turns out that putting $(v-\mu) T^{2}+S=R$ means that $R=R_{1} R_{2}$ (thanks to CAS!) where:

$$
R_{1}=\mu(a-1)^{2}+a b+\mu \nu(a+b-1)
$$

and

$$
\begin{aligned}
R_{2}= & \mu(\nu-\mu)(a-1)^{2}+((\nu-\mu)(1-\mu \nu)+\mu \nu(1+\mu \nu)) a b \\
& +(\mu \nu(\nu+1)+\nu(1-\nu))(b-1)^{2}+(\mu+1-\nu+\mu \nu(\nu+1-\mu))(a+b-1) .
\end{aligned}
$$

$\mathrm{S}_{1}$ and $\mathrm{R}_{1}$ are quadratic expressions which have been arranged in order to show that they are always non-negative when the quadrilateral is convex. $S_{2}$ and $R_{2}$ have been arranged in order to show that they are always non-negative when $\mu \geq v$ and $\mu \leq v$ respectively. Thus, $\rho_{\max 1} \geq \rho$ when $\mu \geq \nu$ and $\rho_{\max 2} \geq \rho$ when $\mu \leq \nu$.

Note that, when $\mu=\nu, S=R$, with $S_{1}=R_{1}$ and $S_{2}=R_{2}$ (to within a scale factor).

Because $S_{1}=0$ and $R_{1}=0$ go through $\mathrm{Z}$ and $\mathrm{X}$ respectively, they account for the zero in the numerator of the difference between the two potential maxima and $\rho$. Curiously, all four conics go through $\mathrm{M}_{\mathrm{XY}}$, while, in the counter-clockwise case, all four go through $\mathrm{M}_{\mathrm{YZ}}$ (Fig. 11).

The same applies to the other orientation where the relevant conics can be found using the interchange.

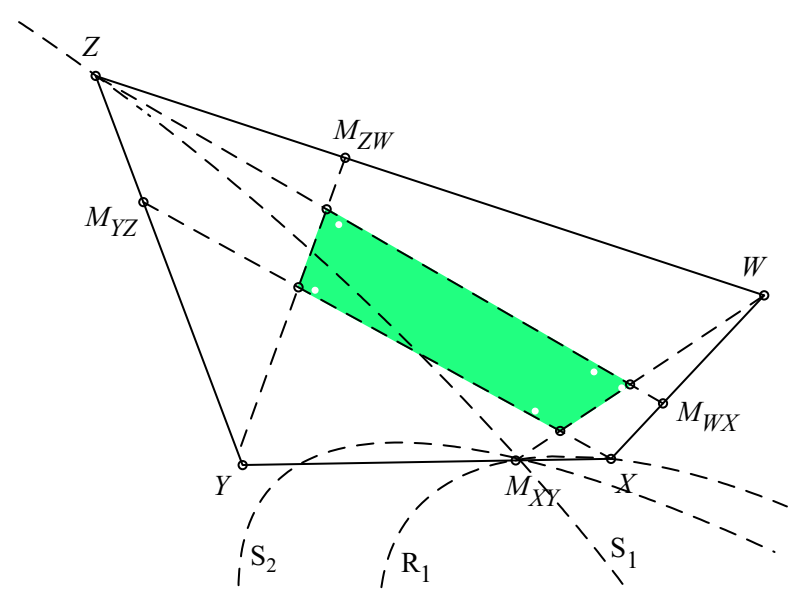

Fig. 11 Conics $S_{1}$ and $S_{2}=0$ on which $\rho_{\max 1}$ is achieved when $\mu>\nu$, one of which passes through $Z$, and conic $R_{1}=0$ on which $\rho_{\max 2}$ is achieved and which passes through $X$ 
It is intriguing, but not particularly edifying, to explore relationships among the individual trapezia conditions and the four conics arising from both of the orientations. For example, $R_{1}-S_{1}=T$ and $S_{2}-R_{2}=(1+\mu v) T$.

\section{Some Historical Remarks}

Exploration began with the special case in which $\mu=\nu=\frac{1}{2}$. Success with proving the extremes of 5:1 and 6:1 generated sufficient confidence to consider the more general case in which the edges are divided in the same ratio cyclically around the quadrilateral. The values for the minimum and maximum were found by guessing that the position of $\mathrm{W}$ would arise in the same way as in the particular case, and so it transpired. The trapezia conditions first emerged from using a CAS to factor the difference between the general ratio and the conjectured minimum. Although obvious in retrospect, there was a moment of excitement when the trapezia conditions were recognised in the numerator of the difference. The format of the denominator of the residual term was discovered only after much gazing and pondering, and a decision to try to make use of the T-expression. When $\mathrm{T}$ was used to eliminate the highest degree terms in $\mu$ appearing in the residual denominator, the rest factored unexpectedly, providing a second frisson of excitement.

Ambition led to trying the most general case in which the ratio divisions on the edges of the quadrilateral are all different, but it soon became evident from the DGS (and quiet reflection) that there could not be any satisfying extremes for the ratio.

Embarking on dividing opposite edges in the same ratio, using $\mu$ and $\nu$, began easily enough. Subtracting the conjectured minimum achieved with a parallelogram, then factoring, revealed the trapezium conditions as expected. However, it was a challenge to deal with the two possible maxima. It was only in retrospect when revising these notes that the idea of relating the numerators of the differences $\rho-\rho_{\min }$ and $\rho_{\max 1}-\rho$ made it obvious how to find the residual denominators. Whenever the algebra became an obstacle, use was made of specific examples to check comprehension of the algebra, geometrically.

Showing that the $S$ and $R$ expressions were non-negative took even more gazing and pondering until the formats used here were discovered. They were then checked by constructing a procedure to re-express quadratic expressions in $a$ and $b$ in terms of ( $a-$ $1)^{2},(b-1)^{2}, a b$ and $(a+b-1)$. It was startling to discover these terms as a basis for presenting the constraints. They provide the reason for presenting the trapezia conditions in the form used. Of course, the $a b$ term can be re-written as $(a-1)(b-1)$, with a revision to the coefficient of $a+b-1$ so as to express the conics neatly, but the present form is needed in order to see directly that the quadratic expressions are always nonnegative when the quadrilateral is convex.

\section{Special Case of Trapezia}

Consider the case in which $z m w x$ is parallel to $x m y z$, so that $\mu(a-1)+(b-1)=0$. The parallel lines have implications for areas as shown in Fig. 12 (left). 
Drawing a line through $\mathrm{M}_{\mathrm{ZW}}$ parallel to $w m x y$ and a line through $\mathrm{M}_{\mathrm{XY}}$ parallel to $y m z w$ creates two triangles (shaded in Fig. 12, right side). Denote their areas by $\Delta_{C}$ and $\Delta_{D}$ respectively. Then:

$$
\left(\frac{1}{v^{2}}-1\right)\left(C_{1}+\Delta_{C}\right)=C_{2}+\Delta_{C} \text { and }\left(\frac{1}{v^{2}}-1\right)\left(D_{1}-\Delta_{D}\right)=D_{2}-\Delta_{D}
$$

using the parallels construction making up the dark-shaded triangles.

Reading around the inner quadrilateral, Area $_{\text {outer }}=$ Area $_{\text {inner }}+\frac{A+B}{\mu^{2}}+C_{1}+C_{2}+D_{1}+D_{2}$.

Considering the four triangles that surround the inner quadrilateral, but with overlaps,

$$
\text { vArea }_{\text {outer }}=\frac{A}{\mu^{2}}+D_{1}+\frac{B}{\mu^{2}}+C_{1} \text { and } \mu \text { Area }_{\text {outer }}=\left(A+B+C_{1}+D_{1}+C_{2}+D_{2}\right) .
$$

Putting these together, it turns out that:

$\frac{\text { Area }_{\text {outer }}}{\text { Area }_{\text {inner }}}=\frac{1+\mu v}{(1-\mu)(1-v)}+\left(\Delta_{C}-\Delta_{D}\right) K$ where $K$ is some factor.

Since $\Delta_{C}$ and $\Delta_{D}$ are similar, it requires only that they be congruent to validate the minimum ratio. This follows from the algebraic calculation (thanks to a CAS) that:

$$
\frac{V_{1} M_{Z W}}{V_{4} V_{1}}=\frac{\mu^{2} v^{2}}{(1-\mu)^{2}}=\frac{V_{3} M_{X Y}}{V_{2} V_{3}} \text { and } \frac{Y V_{4}}{V_{4} V_{1}}=\frac{\mu^{2}}{(1-\mu)^{2}}=\frac{W V_{2}}{V_{2} V_{3}}
$$

Consequently, $\Delta_{C}=\Delta_{D}$ and so the minimum area ratio is what the algebra says. It remains a challenge to find a synthetic (Euclidean) proof.

\section{Conclusions}

Alerted to an erroneous assumption based on not considering enough decimal places, we were led to an exciting exploration which was made possible for us through the use of a DGS for formulating conjectures, and a CAS for carrying out algebraic calculations with co-ordinates. The two went hand in hand, as we went back and forth between the two making, testing and validating conjectures, while seeking geometrical implications of algebraic relationships.

While synthetic geometry proofs (without using co-ordinates) continue to escape us, combining algebra with geometry provides a rich playground for exploration. Students
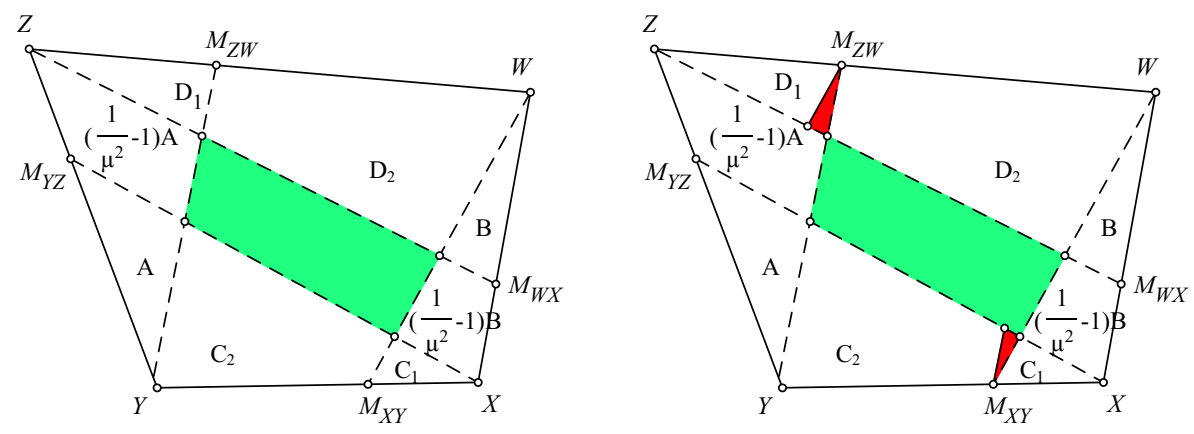

Fig. 12 The trapezium case showing areas and an extra construction 
could be offered the mistaken conjecture that the ratio is always $5: 1$ and invited to try to find the actual minimum and maximum using a DGS alone. Or they could choose values for $\mu$ and $\nu$, and then watch the area ratio change (to two decimal places ... where it remains largely constant) until the they think of increasing the number of decimal places. However, experiencing the power of having a CAS to do the tedious co-ordinate calculations - and relating the geometry to the algebra and vice versa offers an instance of mathematical exploration which would otherwise be too complicated for most students.

Open Access This article is distributed under the terms of the Creative Commons Attribution 4.0 International License (http://creativecommons.org/licenses/by/4.0/), which permits unrestricted use, distribution, and reproduction in any medium, provided you give appropriate credit to the original author(s) and the source, provide a link to the Creative Commons license, and indicate if changes were made.

\section{References}

Bogomolny, A. (2018). The carpet theorem: What is it about? A mathematical droodle. (www.cut-the-knot. org/Curriculum/Geometry/CarpetsInSquare.shtml\#explanation). Accessed March 2019.

Mason. J. (2019). Outer to inner quadrilateral area ratios. (www.pmtheta.com/Outer-Inner-Area-Ratios.html).

Publisher's Note Springer Nature remains neutral with regard to jurisdictional claims in published maps and institutional affiliations. 